\title{
FISH SPECIES USING THE FISH PASSAGE BETWEEN JAMUNA AND BANGALI RIVER AT SARIAKANDI, BOGRA
}

\author{
Zaman, M. and M. N. Naser \\ Department of Zoology, Faculty of Biological Science, University of Dhaka, Dhaka-1000, Bangladesh
}

\begin{abstract}
Sariakandi fish pass is unique connecting Jamuna and Bangali rivers through an engineered channel. A study was conducted on the fish pass aiming to improve fish diversity in north-west Bangladesh. Sampling at the fish pass, market census, interviewing the locals and focus group discussion were done to collect data from the study area. A total of 69 fish species under 9 orders and 26 families were recorded. Cypriniformes (22 species) was the most dominant order followed by Siluriformes (21 species), Perciformes (13 species), Clupeiformes (4 species), Synbranchiformes (4 species), Osteoglossiformes (2 species), Mugiliformes (1 species), Anguilliformes (1 species) and Tetraodontiformes (1 species). In total 30 threatened species in Bangladesh and globally recognized 9 species were recorded. This fish pass is not working during the dry or winter seasons but helps in the diversified fish migration diversity of Bengali and Jamuna river system during the flood time of Bangladesh. The present study recommends establishing effective fish passages in flood protection structures or dams in Bangladesh to retain the connectivity of fish migration routes and the improving of fish diversity.
\end{abstract}

Key words: Fish diversity; Fish pass; Threatened fishes; Bangali River; Jamuna River.

\section{INTRODUCTION}

Sariakandi Fish pass is the largest fish pass of Bangladesh constructed between 1999-2001 connecting the Jamuna river (west bank) and the Bengali river (east bank) at Debdanga of Kutubpur union of Sariakandi Upazilla, Bogra. The fish pass facilities was created by construction of $6 \mathrm{~km}$ embankment to join the Kalitola Groyne with the river bank protection works at Mathurapara. The construction of Brahmaputra-Jamuna right embankment (BRE) in the late 1960 affected the flooding pattern and fish production in the Bangali river and to overcome the effects later structure was built (IUCN 2002). The flood had a major impact on the geography of rivers in the northern region of Bangladesh. The Jamuna and the Teesta were created after a massive flood in 1787. It is speculated that the Bangali River (Fig 1) was born out of the changes of the Jamuna and the Teesta (Chowdhury 2012). The Jamuna and the Bangali River had good numbers of freshwater fishes before implementation of the Brahmaputra right embankment project. After the construction of BRE, fish production in the Bangali river was reduced drastically due to the disruption of the natural fish migration routes between these two rivers. After construction of the fish pass, the water volume and fish species of the Bangali River is increased (Moumita et al. 2011, Zaman et al. 2018).

A fish pass is a hydraulic structure that enables fishes to overcome obstructions in the passage to the spawning grounds and other upstream migration habitats (Bell 1986, Clay 1961). The concept of fish pass was introduced in Bangladesh in the 1990s and since then four fish friendly structures and fish passes have been built (IUCN 2002, Kabir 2010, Zaman et al. 2019). At present, reduction in the abundance of fish species from the inland waters is a burning issue in Bangladesh. The river fisheries of Bangladesh is being deteriorated day by day due to over fishing, construction of flood protection dams, destruction of natural breeding and feeding grounds, use of fertilizer, insecticides and manures etc. Therefore area basis study is required to identify the present status and causes of destruction of faunal diversity in different rivers of Bangladesh. The study was conducted to identify the present status of migratory fishes using the fish passes in flood season from the Bangali to the Jamuna river system. 


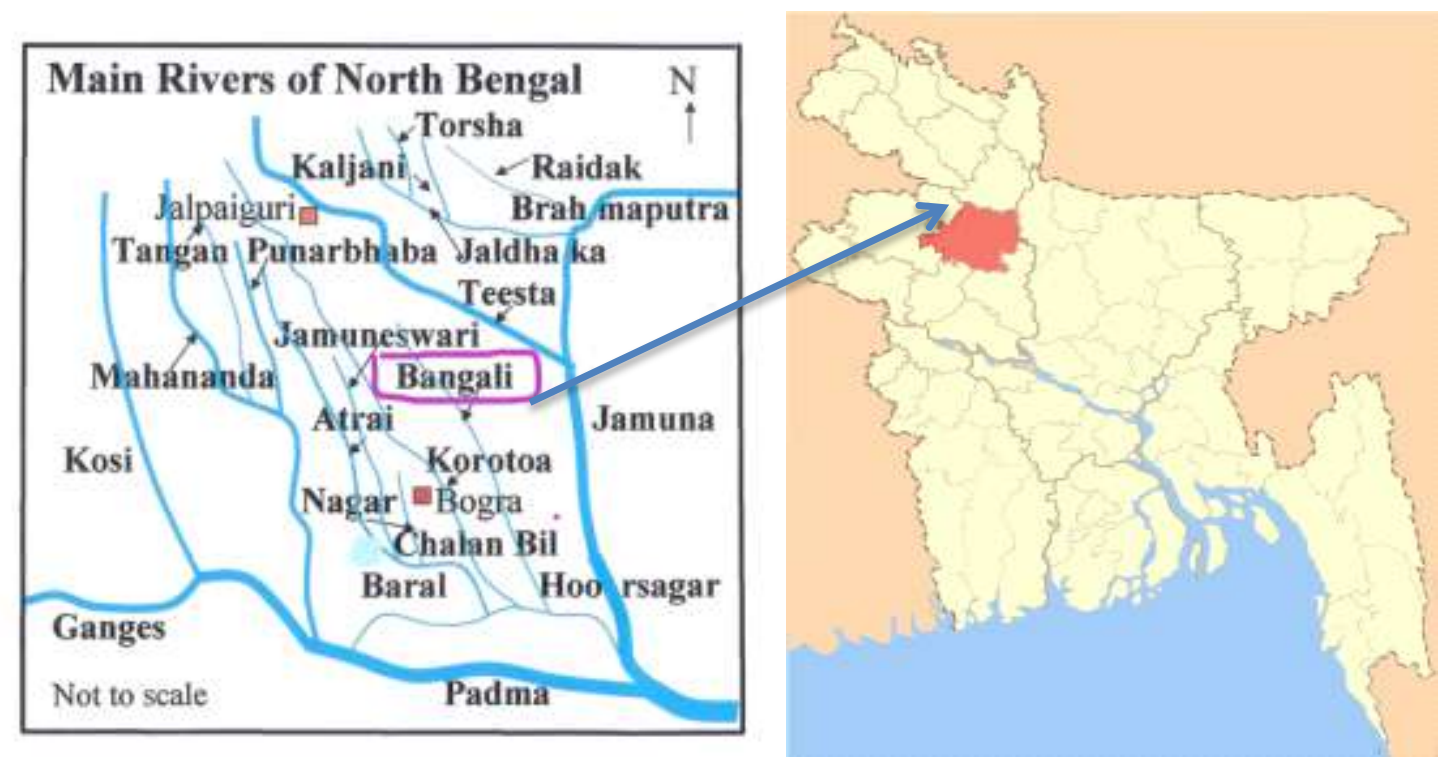

Fig. 1. Map of the Bangali River in Sariakandi, Bogra, Bangladesh.

\section{Study area}

\section{MATERIAL AND METHODS}

The field study was conducted in the Bangali river and the Jamuna river Sariakandi Fishpass at Sariakandi Upazilla, Bogra District, Bangladesh. Located between 24${ }^{\circ} 44^{\prime}$ and $25^{\circ} 03^{\prime} \mathrm{N}$ latitudes and between $89^{\circ} 30^{\prime}$ and $89^{\circ} 45^{\prime}$ E longitudes (Fig 2a). Sariakandi fish pass is the most recent and modern, vertical slot type of fish pass (Fig. 2b), rectangular in shape, length $92.4 \mathrm{~m}$ and width is $15 \mathrm{~m}$. There are 3 separate and parallel passages in the pass (IUCN 2002).

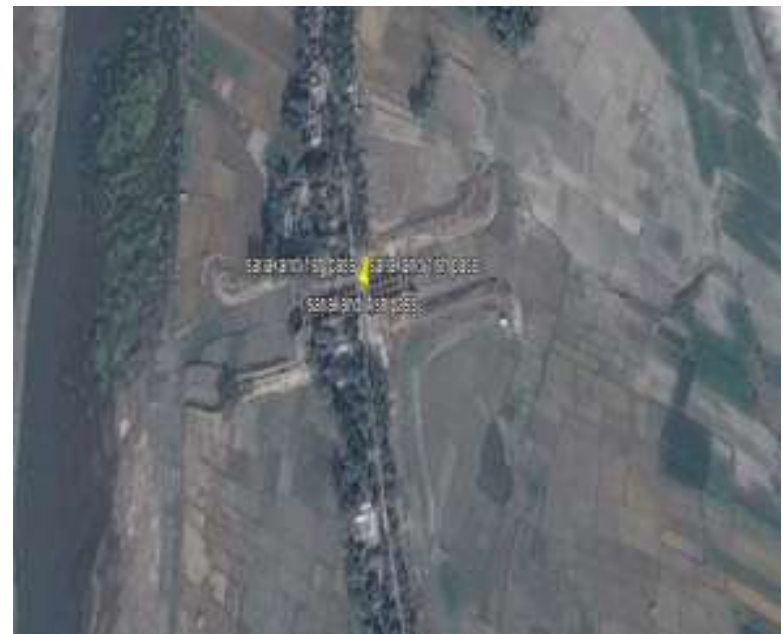

$\mathbf{a}$

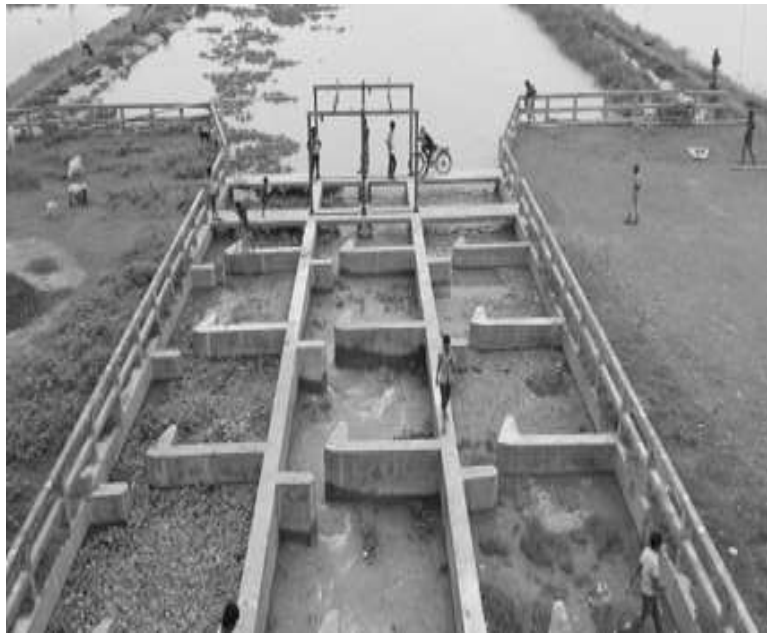

b

Fig. 2. View of the study site: a. Aerial view; b. Vertical slot type fish pass.

\section{Data collection}

Data collection was conducted from May to August 2015. It was attempted with a view to assessing the availability of fishes using the fish pass during migration period. The data collection was made in respect to the fish fauna increase or decline causes created by the fish pass. For this study sampling at the fish pass, local market census, interviewing the inhabitants and focus group discussion were 
considered as the procedural methods. Fish samples were collected from fishermen when on fishing. Fishermen used lift net, cast net, seine net, fish traps dharma jal, khepla jal and ber jal for the catch. Threatened fish samples were collected from the site, preserved in 7-10\% buffered formalin solution for the identification and further study at Bergen Laboratory, Department of Zoology, University of Dhaka.

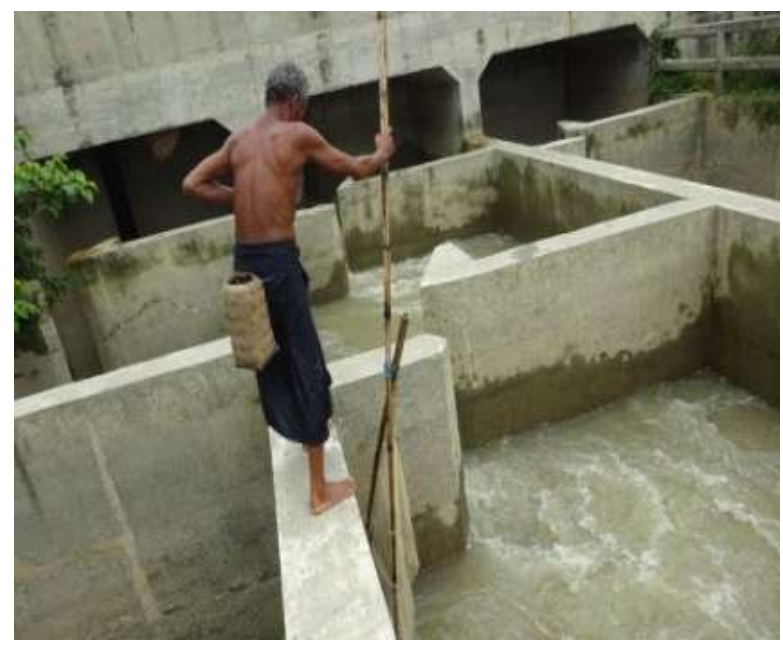

a

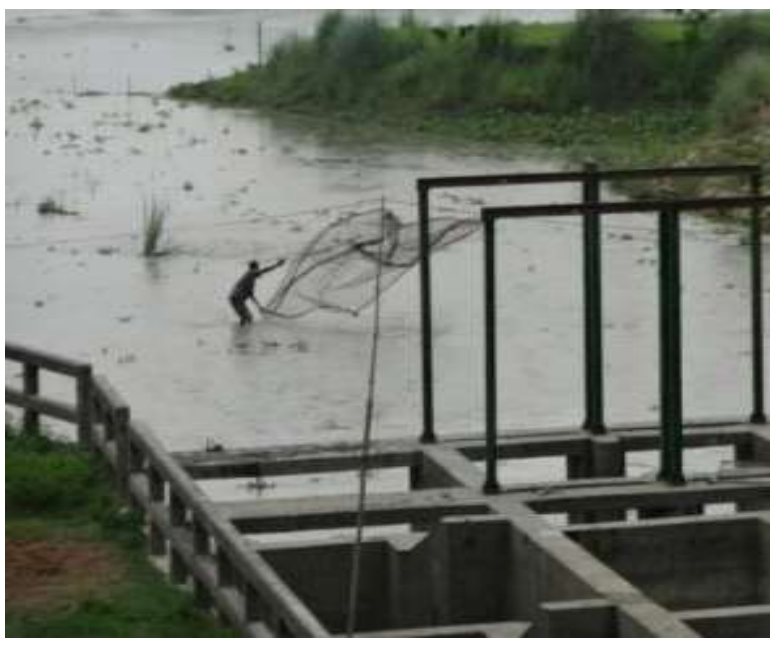

b

Fig. 3. Activities of a fisherman in the fish pass: a. Fisherman on fishing; and b. Using net to catch fish.

To collect data with questionnaire interviews, random sampling method was followed in 50 fishermen from nearby villages adjacent to fish pass. The interview of fishermen was made at home or on fish pass site during fishing (Fig. 3a and 3b). Local market also surveyed for fishes which were available round the year. Focus group discussions (FGD) for fishermen and cross-check interviews with key informants were used. A total of five FGDs were conducted. Each group size of the FGD was 7 to 10 fishermen. Cross-check interviews were made with key person as Upazilla Fisheries Officer. Water quality parameter data were collected by using Oxygen Meter (Lutron portable dissolved oxygen meter, model no. DO 5509) for oxygen detection, $\mathrm{pH}$ meter (Orion Laboratories, model 210A) for $\mathrm{pH}$ and TDS meter (Ultrapen ${ }^{\mathrm{TM}} \mathrm{PT} 1$ ) used to detect temperature and TDS.

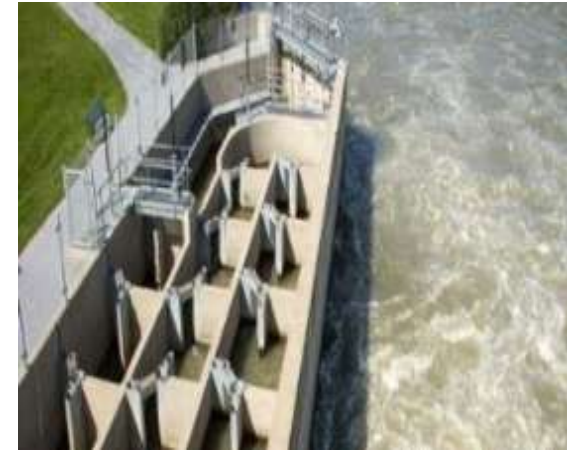

a

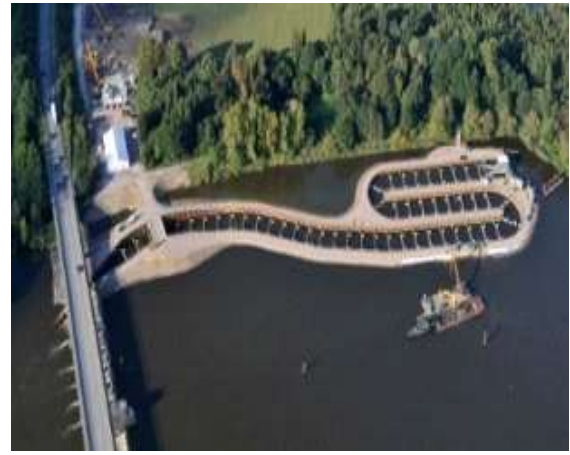

b

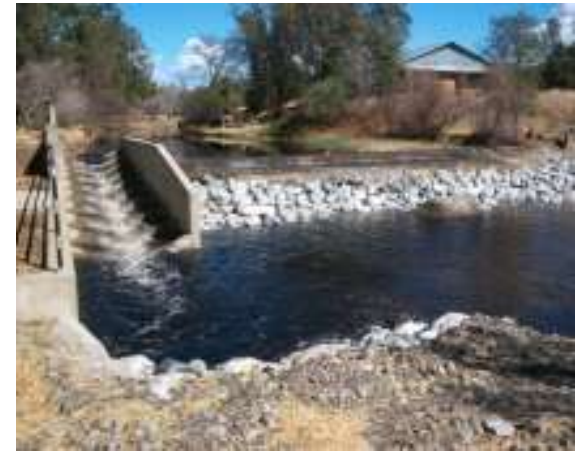

c

Fig. 4. Fish passes in different countries: a. Richelieu River, Quebec, Canada; b. Elbe River, Germany; and c. Millville, California, USA.

Methodology of fish migration study

Fish passes have been developed mainly in North America and Europe for a very limited number of target species, mainly salmonids and clupeids. In these cases, the data is gathered from control station monitoring, trapping or surveillance or some marking, recapture and telemetry methods (Welcomme 
1985). Thiem et al. (2011) made reports on spawning migrations of sturgeon affected by the construction of dams on Richelieu River, Quebec, Canada (Fig. 4a). They found barriers to migration to the imperilment of the sturgeon. Myers et al. (2001) studied ocean distribution and migration patterns of Yukon river chinook salmon. Direct information from high seas tagging studies indicates that Yukon river chinook salmon are concentrated in the Bering Sea. Fish pass designed for Eel and Elver in UK and Europe (Fig. 4b). The aim of the study was to produce design criteria and best practice designs for Eel and Elver passes (Solomon et al. 2004). The Coastal Conservancy (2004) reported inventory of barriers to fish passage in California's coastal watersheds (Fig. 4c), California's salmon, steelhead and other species are vitally dependent on the ecological integrity of dozens of streams and rivers. By following and on understanding the above literature the present attempt has been proceeded to observe the fish migration in the Jamuna river and Bangali river.

\section{RESULTS AND DISCUSSION}

\section{Fish Movement}

Information on fish movement through fish pass was acquired primarily by the fishermen fishing in the study area and secondarily from visual observations. Generally fish species take shelter in the Bangali river during flood recession period. During sampling period presence of these fish species migrating from Bangali river to Jamuna river were collected. The major type of fish species, which moves through the Sariakandi fish pass are listed in the Table 1.

Table 1. Number of collected fishes from the study area

\begin{tabular}{lccccccc}
\hline $\begin{array}{c}\text { No. of } \\
\text { Orders }\end{array}$ & $\begin{array}{c}\text { No. of } \\
\text { Families }\end{array}$ & $\begin{array}{c}\text { No. of } \\
\text { Genus }\end{array}$ & $\begin{array}{c}\text { No. of } \\
\text { Species }\end{array}$ & \multicolumn{2}{c}{$\begin{array}{c}\text { No. of fishes observed } \\
\text { passing fish pass }\end{array}$} & \multicolumn{2}{c}{$\begin{array}{c}\text { No. of fishes not observed } \\
\text { passing fish pass }\end{array}$} \\
\cline { 5 - 8 } & & & & Genus & Species & Genus & Species \\
\hline 9 & 26 & 52 & 69 & 31 & 35 & 27 & 34 \\
\hline
\end{tabular}

Type of fishes

A total of 69 species of fish belonging to 26 families of nine orders were recorded. Observation states that 51 genera contain the examined 69 species. Among them 35 fish species under 31 genera were observed crossing the fish pass. Fishermen catch showed some threatened fishes.

Table 2. Present status of fish biodiversity in Bangali River after establishment of fish pass

\begin{tabular}{|c|c|c|c|c|c|}
\hline Order & Family & Scientific name & Local name & Status in BD & Global Status \\
\hline \multirow{18}{*}{ Cypriniformes } & \multirow{18}{*}{ Cyprinidae } & Amblypharyngodon mola & Mola, Moa & LC & NE \\
\hline & & Amblypharyngodon microlepis & Mola, Molangi & $\mathrm{LC}$ & $\mathrm{LC}$ \\
\hline & & Systomus sarana & Sarputi & NT & $\mathrm{LC}$ \\
\hline & & Puntius chola & Chola puti & $\mathrm{LC}$ & LC \\
\hline & & Pethia conchonius & Kanchan puti & $\mathrm{LC}$ & LC \\
\hline & & Pethia ticto & Tit-punti & VU & LC \\
\hline & & Puntius sophore & Jat punti, Jati punti & LC & LC \\
\hline & & Aspidoparia morar & Piyali & VU & NE \\
\hline & & Labeo rohita & Rui, Rohu & LC & LC \\
\hline & & Labeo calbasu & Kalibaus & $\mathrm{LC}$ & $\mathrm{LC}$ \\
\hline & & Labeo bata & Bata & $\mathrm{LC}$ & $\mathrm{LC}$ \\
\hline & & Labeo boga & Bhangon, Bata & CR & $\mathrm{LC}$ \\
\hline & & Gibelion catla & Katla, Katol & $\mathrm{LC}$ & NE \\
\hline & & Cirrhinus cirrhosus & Mrigel, Mirka & NT & VU \\
\hline & & Cirrhinus reba & Tatkini, Bata & NT & $\mathrm{LC}$ \\
\hline & & Chela laubuca & Chhep Chela & $\mathrm{LC}$ & NE \\
\hline & & Rasbora daniconius & Darkina & $\mathrm{LC}$ & $\mathrm{LC}$ \\
\hline & & Osteobrama cotio & Dhela & NT & $\mathrm{LC}$ \\
\hline
\end{tabular}




\begin{tabular}{|c|c|c|c|c|c|}
\hline & & Hypopthalmichthys molitrix & Silver carp & \multicolumn{2}{|l|}{ EXOTIC SP. } \\
\hline & \multirow{3}{*}{ Cobitidae } & Botia dario & Rani & $\mathrm{EN}$ & $\mathrm{LC}$ \\
\hline & & Lepidocephalichthys guntea & Puiya, Poa & $\mathrm{LC}$ & $\mathrm{LC}$ \\
\hline & & Canthophrys gongota & Gutum & NT & $\mathrm{LC}$ \\
\hline \multirow{2}{*}{ Clupeiformes } & \multirow[t]{2}{*}{ Clupeidae } & Corica soborna & Kachki & $\mathrm{LC}$ & $\mathrm{LC}$ \\
\hline & & Tenualosa ilisha & Ilish (Juvenile) & $\mathrm{LC}$ & $\mathrm{LC}$ \\
\hline \multirow{15}{*}{ Siluriformes } & \multirow[t]{2}{*}{ Siluridae } & Ompok pabda & Pabda & $\mathrm{EN}$ & NT \\
\hline & & Ompok bimaculatus & Kani pabda & $\mathrm{EN}$ & NT \\
\hline & Pangasidae & Pangasius pangasius & Pangus & $\mathrm{EN}$ & $\mathrm{LC}$ \\
\hline & Heteropneustidae & Heteropneustes fossilis & Shing & $\mathrm{LC}$ & $\mathrm{LC}$ \\
\hline & Claridae & Clarius batrachus & Magur & $\mathrm{LC}$ & $\mathrm{LC}$ \\
\hline & Schilbeidae & Eutropiichthys vacha & Bacha & $\mathrm{LC}$ & $\mathrm{LC}$ \\
\hline & Erethistidae & Conta conta & Bot Tengara & NT & $\mathrm{NE}$ \\
\hline & \multirow{6}{*}{ Bagridae } & Sperata aor & Guji ayr & VU & $\mathrm{LC}$ \\
\hline & & Sperata seenghala & Taila ayr & VU & $\mathrm{LC}$ \\
\hline & & Mystus tengara & Bujuri tengra & $\mathrm{LC}$ & $\mathrm{LC}$ \\
\hline & & Mystus vittatus & Tengra & $\mathrm{LC}$ & $\mathrm{LC}$ \\
\hline & & Mystus cavasius & Kabshi tengra & NT & $\mathrm{LC}$ \\
\hline & & Rita rita & Rita & $\mathrm{EN}$ & $\mathrm{LC}$ \\
\hline & Amblycipitidae & Amblyceps laticeps & Chhota Shingi & VU & $\mathrm{LC}$ \\
\hline & Sisoridae & Bagarius bagarius & Bagha ayr & $\mathrm{CR}$ & NT \\
\hline \multirow{9}{*}{ Perciformes } & Channidae & Channa orientalis & Gachua & $\mathrm{LC}$ & $\mathrm{LC}$ \\
\hline & \multirow{3}{*}{ Ambassidae } & Chanda nama & Chanda & $\mathrm{LC}$ & $\mathrm{LC}$ \\
\hline & & Pseudambassis baculis & Phopha chanda & NT & $\mathrm{LC}$ \\
\hline & & Pseudambassis ranga & Lal chanda & $\mathrm{LC}$ & $\mathrm{LC}$ \\
\hline & Nandidae & Nandus nandus & Bheda & NT & $\mathrm{LC}$ \\
\hline & Anabantidae & Anabas testudineus & Koi & $\mathrm{DD}$ & DD \\
\hline & Osphronamide & Trichogaster fasciata & Kholisha & $\mathrm{LC}$ & $\mathrm{LC}$ \\
\hline & Usphronemidae & Pseudophromenus cupanus & Koi bandi & $\mathrm{LC}$ & $\mathrm{LC}$ \\
\hline & Gobiidae & Glossogobius giuris & Baila, Bele & $\mathrm{LC}$ & $\mathrm{NE}$ \\
\hline \multirow{4}{*}{ Synbranchiformes } & \multirow{3}{*}{ Mastacembelidae } & Mastacembelus armatus & Baim, Gonti & $\mathrm{EN}$ & $\mathrm{NE}$ \\
\hline & & Mastacembelus pancalus & Guchi baim & $\mathrm{LC}$ & $\mathrm{LC}$ \\
\hline & & Macrognathus aral & Tara baim & DD & $\mathrm{LC}$ \\
\hline & Synbranchidae & Monopoterus cuchia & Kuchia & VU & VU \\
\hline Anguilliformes & Ophichthidae & Pisodonophis boro & Bamosh, Kharu & $\mathrm{LC}$ & $\mathrm{LC}$ \\
\hline Tetraodontiformes & Tetraodontidae & Leiodon cutcutia & Potka,Tepa & $\mathrm{LC}$ & $\mathrm{LC}$ \\
\hline
\end{tabular}




\section{Observation of fish migration}

Observation of fish movement from Bangali to Jamuna river was the main objective of Sariakandi fish pass study. Migratory fish species included carps, catfishes, eels, gobies, knife fish, perches, miscellaneous species and prawns. The local fishes categorized into whitefish (carp fish) and blackfishes (catfishes) based on their migratory movements. The white fishes are Rui, Catla, Mrigel, Kalbaush, Gonia, Sarputi etc. of which Rui, Mirgel, Kalbaush are more dominant species than other species. In black fishes are Boal, Pabda, Tengra, Magur, Rita, Ayr, Koi, Shingi, Gulsha, Chital etc. Among them Chital, Gulsha and Rita were more dominant species than other species (Kumar 2016). Scientific names of the fishes are shown in the Tables (Table 2, 3 and 4). Fish fingerling, juvenile and adult movement was observed. From the abundance of species in the catch and the catch frequency related that the Carp fishes (Rui and Catla) are dominant species in the study area, of which Cypriniforme (22 species), Siluriformes (21species), Perciformes (13species), Clupeiformes(4species), Synbranchiformes (4species), Osteoglossiformes(2species), Mugiliformes(1species), Anguilliformes(1species) and Tetraodontiformes (1 species) (Table 2, Fig. 5). Among the recorded families Cyprinidae was the most dominant family with 19 species recorded. In Siluriformes 21 species found from 9 different families.

\section{Threatened fish status}

About 69 species were found using fish pass where 30 are threatened ( 2 critically endangered, 10 endangered, 9 vulnerable and 9 near threatened) (IUCN BD/IOIS status) in Bangladesh. In global perspective 9 species found of which 2 vulnerable, 1 endangered and 6 near threatened fishes. A good number (30) of vulnerable and endangered fishes (Fig. 5) found to be using the fish pass (Table 3).

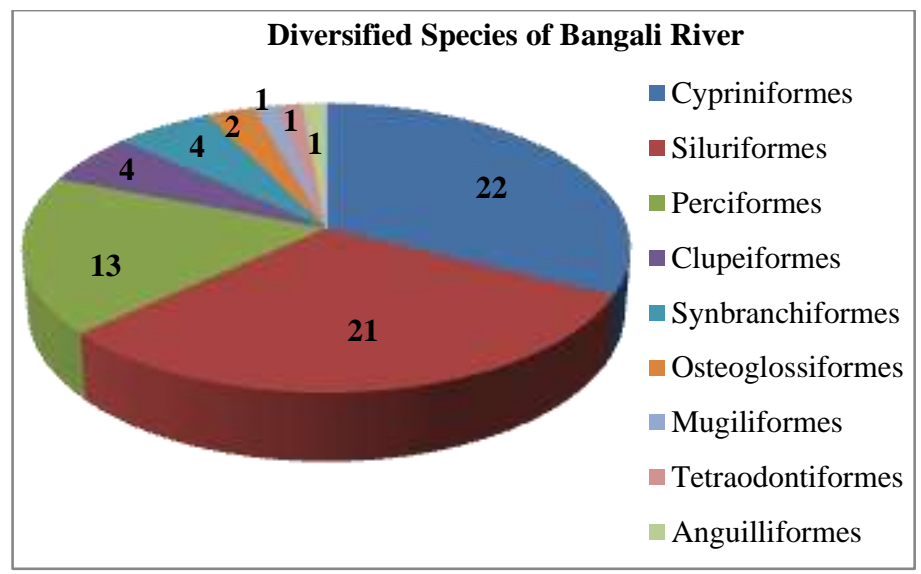

Fig. 5. Composition of diversified species under different orders in the study area

Table 3. Status of threatened fishes using fish pass in Sariakandi, Bogra

\begin{tabular}{lll}
\hline Status & \multicolumn{1}{c}{ Species found in Bangladesh } & \multicolumn{1}{c}{ Globally found Species } \\
\hline Vulnerable (VU) & $\begin{array}{l}\text { Pethia ticto, Aspidoparia morar, Amblyceps laticeps, Gudusia } \\
\text { chapra, Wallago attu, Sperata aor, Sperata seenghala, Notopterus } \\
\end{array}$ & $\begin{array}{l}\text { Cirrhinus cirrhosis, Monopterus } \\
\text { notopterus, Monopterus cuchia, }\end{array}$ \\
\hline Endangered (EN) & $\begin{array}{l}\text { Botia dario, Chela laubuca, Ompok pabda, Ompok bimaculatus, } \\
\text { Pangasius pangasius, Clupisoma garua, Rita rita, Chitala chitala, }\end{array}$ & Conta conta \\
& Channa marulius, Mastacembelus armatus & \\
\hline Near Threatened & Systomus sarana, Cirrhinus cirrhosus, Cirrhina reba, Osteobrama & Wallago attu, Ompok pabda, Ompok \\
(NT) & cotio, Canthophrys gongota, Mystus cavasius, Pseudambassis & bimaculatus, Bagarius bagarius, \\
& baculis, Nandus nandus, Conta conta & Chitala chitala, Ailia coila \\
\hline Critically & Labeo boga, Bagarius bagarius & \\
Endangered (CR) & & \\
\hline Green marked fishes found using the fish pass for migration &
\end{tabular}


Physico-chemical factors in the fish pass

Fish passage success influenced by physico-chemical factors. Field study conducted in Sariakandi at 26.3 to $28.1^{\circ} \mathrm{C}$ temperature, $\mathrm{pH}$ found 8.66 in upstream and 8.50 in downstream. Dissolved oxygen (DO) found to be $5.7-8.13 \mathrm{mg} / \mathrm{L}$ in upstream and 4.9-5.2 $\mathrm{mg} / \mathrm{L}$ in downstream of the water. TDS found ton be 43-46 ppm. Water depth, velocity and flow condition found favorable for fish migration in study site (Biswas 2007).

Table 4. Collected sample fishes habitat and feeding behaviour

\begin{tabular}{|c|c|c|c|c|}
\hline Fish & $\begin{array}{c}\text { Maximum } \\
\text { Length }(\mathrm{cm})\end{array}$ & $\begin{array}{c}\text { Sample } \\
\text { length }(\mathrm{cm})\end{array}$ & $\begin{array}{c}\text { Habitat, abundance, seasonal } \\
\text { availability }\end{array}$ & Feeding habit \\
\hline Rani (Botia dario) & 15.1 & 3.1 & $\begin{array}{c}\text { North Bengal rivers, hill streams of } \\
\text { Chittagong }\end{array}$ & Snail, worm, small shrimp \\
\hline $\begin{array}{l}\text { Lal chanda } \\
\text { (Pseudambassis ranga) }\end{array}$ & 8 & 3 & $\begin{array}{c}\text { Freshwater streams, rivers. Abundant } \\
\text { in rainy season }\end{array}$ & $\begin{array}{l}\text { Zoobenthus, worm, benthic } \\
\text { crustaceans }\end{array}$ \\
\hline $\begin{array}{l}\text { Batashi } \\
\text { (Pseudeutropius atherinoides) }\end{array}$ & 8 & 4.1 & $\begin{array}{l}\text { Amphidromous, breed in mid-May to } \\
\text { July, abundant in Oct-Dec }\end{array}$ & $\begin{array}{c}\text { Algae, plant material, } \\
\text { debris }\end{array}$ \\
\hline $\begin{array}{l}\text { Kholisha } \\
\text { (Trichogaster fasciata) }\end{array}$ & 10 & 4.3 & $\begin{array}{l}\text { Freshwater areas, beels, flood plains, } \\
\text { breed several times on stagnant water } \\
\text { of paddy field during monsoon }\end{array}$ & $\begin{array}{l}\text { Omnivore, feed on insect } \\
\text { larvae. }\end{array}$ \\
\hline $\begin{array}{l}\text { Kaikka } \\
\text { (Xenentodon cancila) }\end{array}$ & 40 & 8 & $\begin{array}{l}\text { Canal, beel, large and medium size } \\
\text { rivers, slow flowing pools. }\end{array}$ & $\begin{array}{l}\text { Predator, live feeder, feeds } \\
\text { on live fish, tadpoles. }\end{array}$ \\
\hline $\begin{array}{l}\text { Koi Bandi } \\
\text { (Pseudophromenus cupanus) }\end{array}$ & 7 & 4 & $\begin{array}{l}\text { Benthopelagic, shallow water, paddy } \\
\text { field, beels. }\end{array}$ & Insects, zooplankton \\
\hline $\begin{array}{l}\text { Bot tengara } \\
(\text { Conta conta })\end{array}$ & 7.8 & 5 & $\begin{array}{c}\text { Rivers of North Bengal, torrential } \\
\text { habitat. }\end{array}$ & Mud and benthic organism \\
\hline $\begin{array}{l}\text { Potka } \\
(\text { Leiodon cutcutia) }\end{array}$ & 13.2 & 3.5 & River, beel, canals and pond & $\begin{array}{c}\text { Shells of molluscs and } \\
\text { crustaceans }\end{array}$ \\
\hline $\begin{array}{l}\text { Silver carp } \\
\text { (Hypopthalmichthys molitrix) }\end{array}$ & 105 & 15 & $\begin{array}{c}\text { Culture fish, naturally occurs in river } \\
\text { system }\end{array}$ & $\begin{array}{l}\text { Phytoplankton, } \\
\text { zooplankton }\end{array}$ \\
\hline Rui (Labeo rohita) & 61 & 9.3 & $\begin{array}{l}\text { Beels, floodplains, clear sluggish } \\
\text { streams, inundated paddy field. } \\
\text { Spawn on May-July in shallow water } \\
\text { or rivers. }\end{array}$ & $\begin{array}{c}\text { Column feeder of mid } \\
\text { water, algae, protozoans, } \\
\text { higher plants, insect larvae, } \\
\text { crustaceans }\end{array}$ \\
\hline $\begin{array}{l}\text { Chhep Chela } \\
\text { (Chela laubuca) }\end{array}$ & 6.7 & 5.1 & $\begin{array}{l}\text { Rivers, canal, ponds. Spawn in } \\
\text { shallow water }\end{array}$ & $\begin{array}{l}\text { Larvivorous, insects, stem } \\
\text { and leaf tissue }\end{array}$ \\
\hline $\begin{array}{l}\text { Kutakanti } \\
(\text { Hara hara })\end{array}$ & 6.2 & 4.5 & $\begin{array}{l}\text { Muddy river bed (Dinajpur, Sylhet, } \\
\text { Mymensign) }\end{array}$ & Benthic organisms \\
\hline $\begin{array}{l}\text { Bamosh } \\
\text { (Pisodonophis boro) }\end{array}$ & 66 & 9 & Shallow beels, mud holes. & $\begin{array}{c}\text { Small fishes, tadpole, } \\
\text { aquatic insects }\end{array}$ \\
\hline $\begin{array}{l}\text { Chhota Shingi } \\
(\text { Amblyceps laticeps })\end{array}$ & 8.5 & 6 & $\begin{array}{c}\text { Dahuki river near Sylhet, Rangpur, } \\
\text { Netrokona }\end{array}$ & $\begin{array}{l}\text { Shells of molluscs and } \\
\text { crustaceans }\end{array}$ \\
\hline $\begin{array}{l}\text { Kajoli } \\
(\text { Ailia coila })\end{array}$ & 15.4 & 5.4 & $\begin{array}{c}\text { Major freshwater rivers and } \\
\text { connected waters } \\
\end{array}$ & $\begin{array}{l}\text { Carnivore, but occasionally } \\
\text { feed algae, debris. }\end{array}$ \\
\hline $\begin{array}{l}\text { Dhela } \\
\text { (Osteobrama cotio) }\end{array}$ & 10.8 & 4 & $\begin{array}{l}\text { Rivers, canals, beels. Abundance } \\
\text { reduced. Breeding starts early } \\
\text { monsoon, continue till September. }\end{array}$ & $\begin{array}{l}\text { Omnivore, surface feeder. } \\
\text { Algae, protozoan, } \\
\text { crustaceans, aquatic insects }\end{array}$ \\
\hline
\end{tabular}

Threatened fish samples were collected from the site, preserved for identification in laboratory (Table 4, Fig. 6). This maiden study of fish fauna recorded a total 69 species including 68 species indigenous and 1 exotic. Fish hatching, juvenile and adult movements were observed in fish pass but maximum fishes were juvenile in size. Fishes were caught when they using fish pass from Bangali River to Jamuna river. The fish size and fish catch indicates that the juvenile species migrates from Bangali 
river to Jamuna mostly. Exotic species (Hypopthalmichthys molitrix) were adult in size, which is popular in Bangladesh, probably escaped from adjacent aquaculture pond during heavy flood. Abundance of threatened fish species among the total catch strongly reflecting its potentiality to be an excellent site for natural conservation. Mass awareness is required for the fishermen to save the threatened fish fauna of the rivers. There should be a strong implementation of conservation laws and acts to make free from illegal catch in fish pass area.

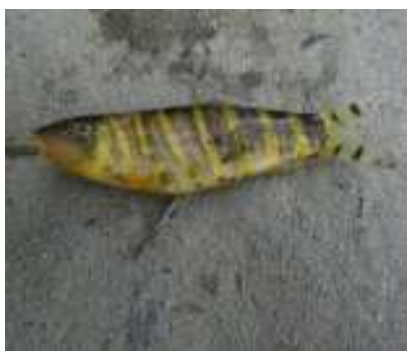

a

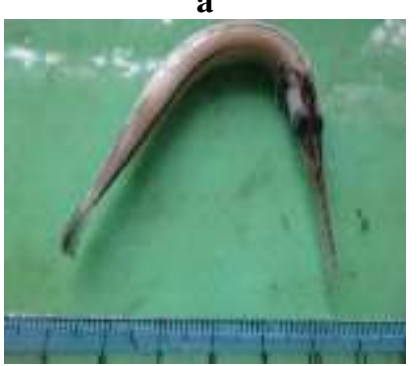

e
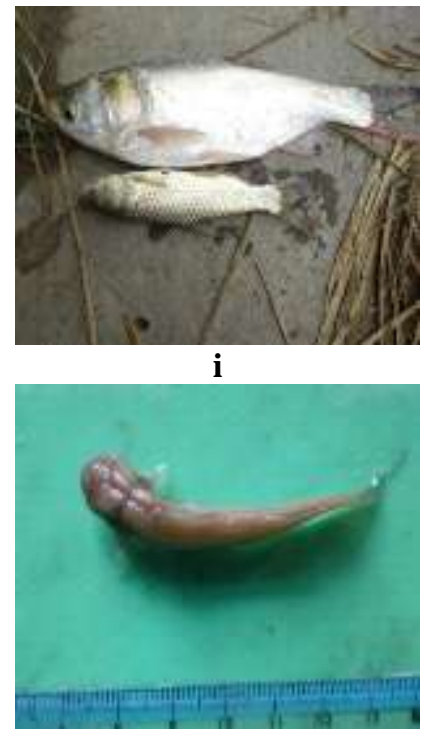

m

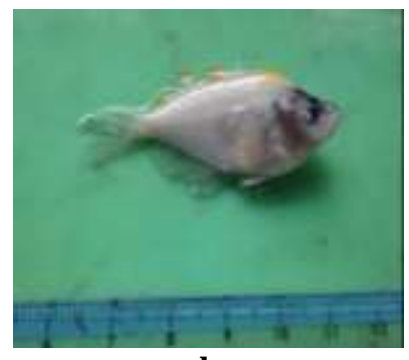

b
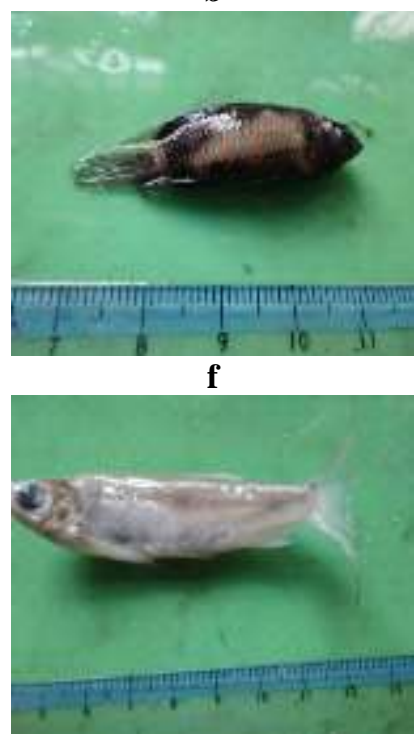

$\mathbf{j}$

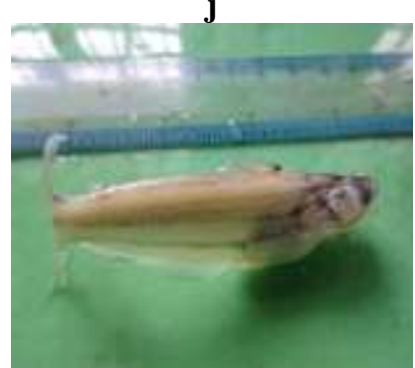

n

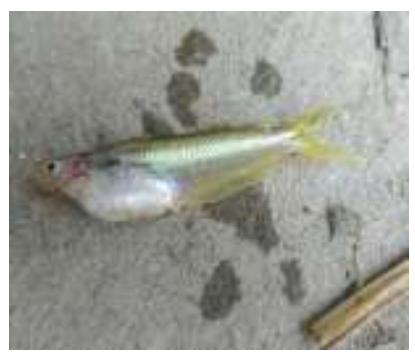

c

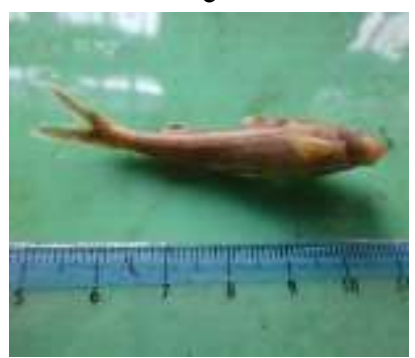

g

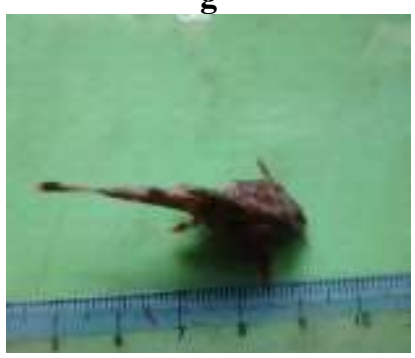

$\mathbf{k}$

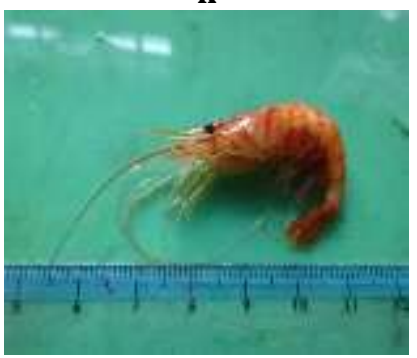

o

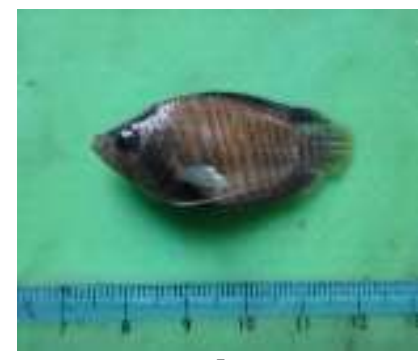

d

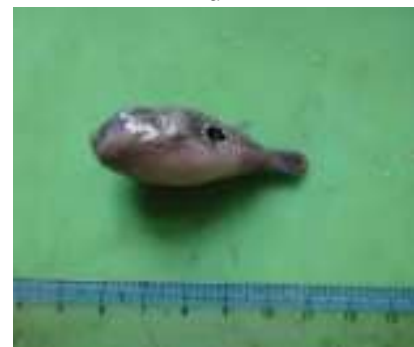

$\mathbf{h}$
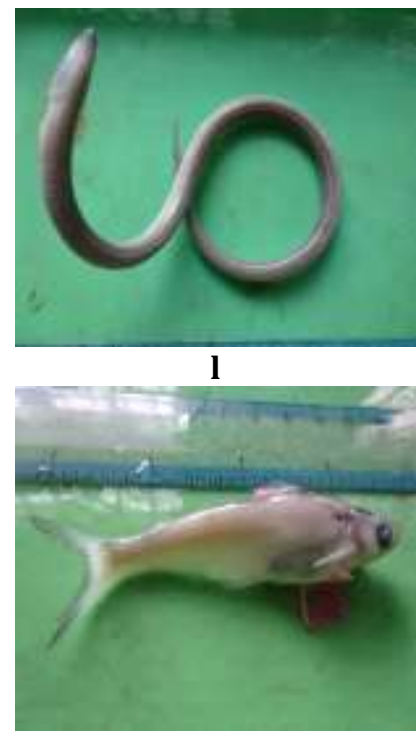

p

Fig. 6. Fishes found using the fish pass: a. Rani (Botia Dario); b. Chanda (Pseudambassis ranga); c. Batashi (Pseudeutropius atherinoides); d. Kholisha (Trichogaster fasciata); e. Kaikka (Xenentodon cancila); f. Koi Bandi (Pseudophromenus cupanus); g. Bot Tengara (Conta conta); h. Potka (Leiodon cutcutia) i. Silver carp (Hypopthalmichthys molitrix) and Rui (Labeo rohita); j. Chhep Chela (Chela laubuca); k. Kutakanti (Hara hara); 1. Bamosh (Pisodonophis boro); $\mathrm{m}$. Chhota Shingi (Amblyceps laticeps) n. Kajoli (Ailia coila) o. Chingri (Insects); and p. Dhela (Osteobrama cotio)

A range of studies have been documented that their efficiency of fish passes can be highly variable (Marmulla and Larinier 2011). Sturgeon spawning migration on Richelieu river, Quebec, Canada fishway resulted $82.2 \%$ (88 of 107) fishes passing through fish pass. The remaining individuals either 
made no attempt. Which indicate successful ascension occurred (Thiem et al. 2011).Restoring connection of riverine systems that have been fragmented by dams is a critical step towards rebuilding fish population and preventing extinction. Fish ways are regarded as potential strategy for mitigating the installation of barriers.

Fish pass between Jamuna and Bangali river study shows that fish way has a positive impact on fish migration. Resulting an improved livelihood of the rural people living at the adjacent villages around the fish pass. After construction of the fish pass, the water volume and fish species of the Bengali River is increased along with the fish diversity and fish biomass. Study showed a good number of threatened species taking the fish pass which indicates faunal exchange between the rivers through fish pass. Water parameters, depth, velocity and flow were also favourable for fishes. Study showed in 69 species, 30 threatened species (IUCN, BD 2015) passing fish pass where 9 species are globally threatened, which may extinct in near future due to improper management. From this study it is evident that fish pass aided in improving the fish diversity in Jamuna river by adding fish from Bangali river system. Further study on the aspect could enlight more information of fish migration habits.

\section{ACKNOWLEDGEMENTS}

This study was the part of the Ph.D. research of M. Zaman under supervision of Professor Dr. M. N. Naser. MZ received the prestigious scholarships from the Prime Minister's Office for this work. In addition, research fund received from the Center for Environment and Geographic Information Services (CEGIS) Bangladesh.

\section{REFERENCES}

Bell, M. C. 1986. Fisheries handbook of engineering requirements and biological criteria. US Army Corps of Engineers, North Pacific Division, Portland., pp. 231-273.

Biswas, R. K. 2007. Study of the Operational Management of Sariakandi Fishpass. M. Sc Thesis, Department of Water Resources Engineering, BUET, Dhaka. 44 pp.

Chowdhury, M. H. 2012. Bangali River. Banglapedia: National Encyclopedia of Bangladesh. 2nd ed. Asiatic Society of Bangladesh. 5 pp.

Clay, C. H. 1961. Design of Fishways and Other Fish Facilities. Dept. of Fisheries of Canada, Ottawa, Canada. 301 pp.

Encyclopedia. 2007. Freshwater Fishes of Bangladesh. Vol 23. Asiatic Society of Bangladesh. 251 pp.

IUCN. 2002. Fish Pass and Fish Friendly Regulators Study. Final Report. Fourth Fisheries Project, Department of Fisheries, Government of Bangladesh., pp. 7-79.

IUCN Bangladesh. 2015. Red Book of Threatened Fishes of Bangladesh. IUCN-The World Conservation Union, Dhaka, Bangladesh., pp. 49-314.

Kabir, S. M. H. 2010. Sariakandi Fish Pass: A Modern Fish Friendly Structure of Bangladesh. Global Warming Bangladesh Fisheries Convention- 2009. Department of Zoology, Rajshahi University., pp. 1-3.

Kumar, B. 2016. Hydraulic Impact on Fish Migration in Sariakandi, Fish Pass of Bangladesh. International Conference and Ecohyhydrology for Fish Passage. 2: 19.

Marmulla, G. and M. Larinier, 2011. Fish Passes: Types, Principles and Geographical Distribution an Overview. Proceedings of the second international symposium on the Fish Pass, FAO. VialedelleTerme di Caracacalla, Roma, Italy., pp. 1-22. 
Moumita, D., M. A. Hussain, M. M. Alam, A. G. Mazlan, and K. D. Simon, 2011. Impact of Sariakandi fish pass on fisheries diversity of Bangali river, Bogra, Bangladesh. AACL Bioflux. 4(5): 621-626.

Myers, K. W., R. V. Walker and N. D. Davis, 2001. Ocean Distribution and Migration Patterns of Yukon River Chinook Salmon. High Seas Salmon Research Program, SAFS-UW-0109. 11 pp.

Solomon, D. J. and M. H. Beach. 2004. Fish Pass design for Eel and Elver (Anguilla anguilla). Environment Agency, R\&D Technical Report W2-070/TR., pp. 19-24.

The Coastal Conservancy. 2004. Inventory of Barriers to Fish Passage in California's Coastal Watersheds. State Coastal Conservancy Report, Oakland, CA 94612.91 pp.

Thiem, J. D., T. R. Binder, J. W. Dawson, P. Dumont, D. Hatin, C. Katopodis, D. Z. Zhu and S. J. Cooke. 2011. Behaviour and passage success of upriver-migrating lake sturgeon Acipenser fulvescens in a vertical slot fishway on the Richelieu River, Quebec, Canada. Endang Species Res. 15: $1-11$.

Rahman, A. K. A. 2005. Freshwater Fishes of Bangladesh. 2nd ed. Zoological society of Bangladesh. 394 pp.

Welcomme, R. L. 1985. River Fishes, FAO Fish, Technical Bulletin Paper. 358 pp.

Zaman, M. and M. N. Naser. 2018. Fish Biodiversity and Migration in Sariakandi Fish Pass of Bangladesh. BAPA-BEN. Pp. 172-179.

Zaman, M., U. K. Navera, A. Hassan and M. N. Naser. 2019. Effective Fish Pass: An Environmental Friendly Approach for Bangladesh Flood Control and Road Infrastructure. FANBangladesh. 5: 12-14. 